\title{
CONTINUOUS FLOWS WITH CLOSED ORBITS ${ }^{1}$
}

\author{
BY ANATOLE BECK
}

Communicated by R. H. Bing, April 4, 1960

Introduction. A continuous one-dimensional flow (hereinafter called simply a flow) in a topological space $X$ is a continuous mapping from $R \times X$ onto $X$ ( $R$ denotes the real line) which has the group property:

$$
\phi(a, \phi(b, x))=\phi(a+b, x), \quad \text { for all } a, b \in R, x \in X .
$$

Each point has an orbit $\vartheta(x)=\{\phi(a, x), a \in R\}$. We are especially interested in the set of points whose orbits consist of single points, that is, those fixed under the flow:

$$
\phi(a, x)=x, \quad \text { all } a \in R .
$$

This set of fixed points is called the invariant set of $\phi$, and in this note, we announce a topological characterization of those sets which are the invariant sets of flows in the Euclidean plane all of whose orbits are closed.

1. We first turn our attention to the flows with compact orbits. Since an orbit can only be a point, a simple closed curve, or a simple arc without its endpoints, this hypothesis restricts us to flows whose orbits are points (the invariant set) and simple closed curves. It is easily seen that the invariant set is closed, and as a result, the intuition fixes at once on the conjecture that the points not invariant under $\phi$ constitute a family (necessarily countable) of disjoint open annuli (an annulus here will mean a homeomorph of the open set of points between two concentric circles). This latter takes some proof, mostly attention to details, but a few lemmas (whose content is visually obvious) suffice to give us

THEOREM 1. If $\phi$ is a continuous flow in the (Euclidean) plane and if all the orbits of $\phi$ are compact, then the invariant set of $\phi$ is the complement of a (countable) disjoint union of open annuli.

We are interested in the converse of this theorem. It is not hard to see that given any annulus, it is possible to construct a flow which leaves no point of the annulus fixed, has compact orbits, and extends continuously to the boundary where the flow has fixed points on the whole boundary. In fact, this can even be accomplished under the

1 The research for this paper was done under a grant from the Wisconsin Alumni Research Foundation. The author wishes to express his debt to Professor Deane Montgomery for his encouragement and helpful suggestions. 
additional requirement that the orbits be differentiable curves, or polygons, or the analytic images of circles. Then, for any set $G$ which is the disjoint union of open annuli, we can carry out this task in each annulus of $G$, and, defining the flow to be fixed at each point of $G^{\prime}$, we obtain

Theorem 2. If $G$ is a disjoint union of open annuli in the plane, then there is a continuous flow in the plane whose invariant set is $G^{\prime}$.

Let us examine the set $F$ of points indicated in Theorem 1. It is closed, so that each component is closed. If any of the components of $F$ are unbounded, then the union of these components is a closed set, which we will call $F_{\infty}$. The removal of $F_{\infty}$ from the plane leaves a (finite or infinite) countable collection of 2 -cells, ${ }^{2}$ and in each of these, the invariant set of $\phi$ is closed, and has only compact components. This leads us to

Definition 1. Let $C$ be a 2-cell, and let $F$ be a closed subset of $C$ which is the complement of a union of pairwise disjoint open annuli lying in $C$. Then if every component of $F$ is compact, $F$ is called a $T$-set of $C$.

TheOREM 3. Let $\left\{C_{i}\right\}$ be a finite or infinite sequence of pairwise disjoint 2-cells in the plane and let $F_{i} \subset C_{i}$ be a $T$-set of $C_{i}, i=1,2, \ldots$. Then the set

$$
F=\left(\bigcup_{i} F_{i}\right) \cup\left(\bigcup_{i} C_{i}\right)^{\prime}
$$

is the invariant set of a flow with compact orbits, and every such invariant set can be so represented.

We also see that if the flow be further restricted to have orbits which are differentiable curves, or polygons, or analytic images of circles, the set of invariant points has the same characterization.

2. In the case of a flow with closed orbits, those orbits which are simple arcs have their endpoints at $\infty$. It can be shown that if $\phi$ is such a flow, and if $x$ is in the invariant set $F$ of $\phi$, and if $x$ is also the limit point of a sequence $\left\{x_{i}\right\}$ of points each of which has a noncompact orbit, then the component of $x$ in the invariant set is unbounded. Furthermore, if $F_{\infty}$ is removed from the plane, we can then prove that the set of points whose orbits are not compact is closed in the set which remains. Removing this set from the plane also, we have the remaining set a union of disjoint 2-cells in each of which the flow has only compact orbits. It follows immediately that in each such

\footnotetext{
${ }^{2}$ Here, and throughout this note, "2-cells" are taken to be open 2-cells.
} 
cell, the invariant set of $\phi$ is a $T$-set, and that the totality of invariant points not contained in $F_{\infty}$ is included in this way.

Definition 2. Let $G$ be a 2-cell, and let $\left\{C_{i}\right\}$ be a finite or infinite sequence of pairwise disjoint 2-cells contained in $G$. Let $F_{i} \subset C_{i}$ be a $T$-set of $C_{i}, i=1,2, \cdots$. Then if $U_{i} F_{i}=F$ is closed in $G$, we say that $F$ is a $T_{\sigma}$-set of $G$.

REMARK. A $T_{\sigma}$-set may be empty. A $T$-set cannot.

Now let $F$ be an invariant set of a flow $\phi$ with closed orbits. Let $F_{\infty}$ be the set of points of $F$ whose components in $F$ are unbounded. Then $E^{2}-F_{\infty}$ is a disjoint union of 2-cells. If $G_{i}$ is one of these 2-cells, and $G_{i}$ is bounded, then the flow $\phi$ in $G_{i}$ has only compact orbits, so that $F \cap G_{i}$ is a $T$-set of $G_{i}$. If $G_{i}$ is unbounded, then the removal of the points with noncompact orbits may leave nothing in $G_{i}$, or may leave a union of finitely many or infinitely many disjoint 2-cells. If there are some 2-cells left by this procedure, then in each of them, $C_{i, j}, F \cap C_{i, j}$ is a $T$-set of $C_{i, j}$. Since $F \cap G_{i}$ must be closed in $G_{i}$, and $F \cap G_{i}=U_{j} F \cap C_{i, j}, F \cap G_{i}$ is a $T_{\sigma}$-set of $G_{i}$.

Now let us examine the converse of this proposition. Suppose $F$ is a closed set. Then the removal of $F_{\infty}$ leaves in the plane a (finite or infinite) countable disjoint union $U_{i} G_{i}$ of 2-cells. Suppose that in each bounded 2-cell $F$ is a $T$-set, and in each unbounded one, $F$ is a $T_{\sigma}$-set. We already know that we can define a flow in each bounded $G_{i}$ which gives $F \cap G_{i}$ as the invariant set, has only compact orbits, and extends continuously to the fixed points on the boundary. We can also show that any $T_{\sigma}$-set in an unbounded $G_{i}$ can be similarly represented, with orbits which are either compact or lines with endpoints at $\infty$. This result gives us

THEOREM 4. Let $\left\{G_{i}\right\}$ be a finite or infinite sequence of pairwise disjoint 2-cells. Let $F_{i} \subset G_{i}$ be a $T$-set of $G_{i}$ if $G_{i}$ is bounded, and a $T_{\sigma}$-set of $G_{i}$ if $G_{i}$ is unbounded, $i=1,2, \ldots$. Then the set

$$
F=\left(\bigcup_{i} F_{i}\right) \cup\left(\bigcup_{i} G_{i}\right)^{\prime}
$$

is the invariant set of a flow with closed orbits, and every invariant set of such a flow can be so represented.

As before, the restriction of orbits to differentiable curves, polygonal arcs, or analytic images of circles or arcs of circles do not affect the characterization.

Corollary (to TheOREM 4). The points of a convergent sequence together with the limit, is not an invariant set of a flow with closed orbits if the sequence has infinitely many distinct terms.

UNIVERSITY OF WisCONSIN 